\title{
Digital transformation and its challenges to the strategic management system
}

\author{
Irina Maksimenko*, Tatiana Vashko, and Svetlana Zdrestova-Zakharenkova \\ Siberian Federal University, 660041 Krasnoyarsk, Russia
}

\begin{abstract}
Digital technologies and their tools are fundamentally changing not only the technological order, but also the economic system. Recently, researchers and the business community attention has been focused on digital companies born in the era of the modern Internet and offering value propositions based on digital technologies and their cross-border properties. But how multifaceted and heterogeneous the nature of the technologies is, so complex and multidimensional is the process of their implementation, which transforms not only the company's IT environment, but also the whole strategic management system. In the process of this transformation, companies face a range of challenges that can lead to devastating consequences. Understanding the sources and nature of these challenges allows to find ways to overcome them and transform them into competitive advantages. The structure of the article includes two blocks. The first block summarizes evolutionary ideas about transformation through its main constructs, parameters, and stages. The second block shows the study and systematization of the challenges that lead the waves of digital transformation in the context of their impact on the parameters of the strategic management system.
\end{abstract}

\section{Introduction}

Digital transformation (DT) is becoming a major topic for companies around the world $[1,2,3]$. Companies that can't adapt to the digital world are expected to become victims of 'Digital Darwinism', where incumbents can disappear and only the most adaptable companies, responding to technological trends, will survive to stay in the market, changing its competitive landscape [4].

By now, most of the companies are involved in DT. Deloitte research showed that $87 \%$ of executives surveyed consider DT to be an important strategic objective for their business [5]. However, when rapid evolution is required, the DT perspective is often accompanied by many challenges, based on the realization that digital transformation is a destructive process that often throws companies into uncharted territory for their business. A complete DT

\footnotetext{
* Corresponding author: iriva16@mail.ru
} 
usually leads to new and unfamiliar operational processes and can change entire business models.

McKinsey's years of research into DT processes have shown that the success rate of these efforts is consistently low: less than $30 \%$ success rate. The results of the pandemic 2020 suggest that digital transformation has become even more difficult. Only $16 \%$ of respondents said that digitalization in their companies has successfully increased productivity and prepared them to support change in the long term. Another 7\% say productivity has got better, but these improvements have not been saved. Even digital-oriented industries such as IT, media and telecommunications are having a hard time. Among these industries, the success rate does not exceed 26\%. But in more traditional industries such as infrastructure, pharmaceuticals, services, digital transformation is even more difficult: success rates fall from $4 \%$ to $11 \%$ [6].

\section{Study methodology}

The research presented in this article is based on a comprehensive and thorough assessment and review of existing research from different professional sources. It includes the main ideas of specialized publications, scientific articles and expert opinions from a number of scientists, research companies and professional associations. In order to build a holistic picture of the modern research landscape, more than 35 publications were reviewed, processed and analyzed. In addition, expert interviews were taken into account to draw up a comprehensive paradigm of digital transformation challenges to the company's strategic management system.

The results of this article are intended to form the basis for further research in this area in order to expand the scope of scientific discussions on the impact of DT on modern models and tools of strategic management. Therefore, structuring the main challenges of digitalization in the form of constructs map shall be riched with empirical studies of the degree of their destructive power for companies in different industries.

\section{Discussion of results}

\subsection{Evolutionary development of digital transformation}

DT is a rather fragmented field from an academic point of view. It is the result of numerous and varied research areas such as the digital transformation of societies, industries, economies and individuals. Recent works related to DT have generally focused on studying its problems, driving forces and failures. Although the key role of a specialized transformation strategy has already been highlighted in the literature, it is still in its infancy, requiring deeper study to understand the transformation roadmap [7, 8, 9]. Therefore, studying DT from a strategic point of view should complete the academic literature with valuable ideas, as well as help the top management of companies in understanding both the DT processes themselves and the underlying building blocks of this transformation [10,11].

It can be argued that society and the economy have reached the peak of the industrial revolution, which bases on the experience of previous ones, but uses new digital technologies with 'full force', as a result of which both the development and the diffusion of innovations occur much faster than before. The new global world economy is characterized by dynamism, individualization and intensity of competition, and the cornerstone of success in it's the introduction of knowledge, technology and innovation in products and services [12].

The need for transformation is also a clear business reality that appears across all industries and affects companies of all sizes and types [13]. It is not surprising that most 
companies expect IT and digital technologies to contribute more and more to the strategic development of their business in the next decade [8].

The roots of digital transformation can be traced back to the 1990s, when 'transformation' became a catchphrase for a variety of practices and organizational outcomes. D. Muzyuka defines the concept of 'transformation' through four parameters [14]:

- Reengineering: increasing organizational efficiency while simultaneously solving the problem of partially increasing labor force involvement;

- Restructuring: increasing efficiency without necessarily improving the organizational ability to achieve their long-term goals and seize opportunities;

- Renewal: increasing efficiency, effectiveness and innovation by empowering employees without a clear focus on desired results;

- Regeneration: improving of existing processes and fundamentally revising the portfolio of existing capabilities.

Business transformation was first linked to the area of strategic management in the $1990 \mathrm{~s}$ K. Prakhalad and A. Osterfeld [15], described it as the generation of strategies and management processes that shall be managed by new ideas, a new concept of opportunities.

Already in the early 2000s, many authors set the rules and criteria for transformation that correspond to IT technologies. For example, V. Richardson et al. [16] believed that information technology is a transformational technology if it fundamentally changes traditional ways of business by redefining capabilities, processes and relationships. They should also include strategic obtaining to develop new opportunities or enter a new market. Finally, IT usage should fundamentally change the way of performing tasks and enable firms to operate in different conditions and in different markets, serve different customers, and finally take a significant competitive advantage. Like business transformations, F. Julliart and J. Kelly [17] classified IT transformations into four constructs:

- Rethinking the company's view of itself and the business understanding;

- The company internal configuration restructuring for the company flexibility;

- Activating the company's activity to bring its value chain-let according to market opportunities;

- Personnel updating in way to professional development.

In an attempt to combine the work of several authors on IT transformation, R. Morgan and K. Page [18] suggest four stages of companies progress. They build on each other and increase risk and reward as companies move through them. These stages include:

- Adaptation, when certain types of activities shall be automated;

- Evolution, when ICT alignment shall be created;

- Foresight, when the business network process shall be rebuilt;

- Renewal, when the field of activity shall be reformulated.

Later, numerous ideas about digital transformation began to appear in the literature. The digital transformation idea appears from a mixture of personal and corporate IT environments and encapsulates the transformational effect of new digital technologies [1, 20].

Thus, the information revolution has recognized the strategic importance of information systems integration in order for companies to obtain and maintain competitive advantages $[21,22]$. The internal business environment of the companies has been focused on cost savings, operational efficiencies and strategic differentiation from competitors. Externally, companies have reaped the benefits of the increased cost and productivity of technology, which has led to the appearance of new products and services based on information technology and systems.

Many aspects of DT are revealed when trying to uncover the context of the cause of transformation, and the latter can be divided into internal motives and external triggers.

Internally, companies are motivated to transform as a result of reduced sales and financial pressure on the current business [8]. They also try to provide social and economic benefits to 
their stakeholders, with a focus on their employees, who actively seek to communicate and collaborate more effectively with the customers they serve. Today, employees want to work in digital companies and demand better IT technologies and more flexibility in their work environment $[1,2,24]$. Operational factors are also linked with the digital transformation urge against the backdrop of expected efficiency gains [23] (or closely related productivity gains [12]).

Externally, digital technology plays a key role as a trigger for transformation. In particular, their speed of development [25], their marketing changes [8, 26] and the destructive potential of the industry [3,27], require companies quickly reaction and accumulation their digital resources. Due to the emerging integration of mobility and social media, tech-savvy clients have completely changed their behavior and expectations of the companies they interact. More importantly, they expect that companies not only respond to their requirements but also anticipate their future needs before customers define them. Besides the customers, the general pressure is also coming from increased digital competition in a highly globalized world, which is another reason for companies to accelerate their transformation efforts [25, 27].

In general, most researchers conclude that digital transformation is a more complex type of business transformation, which shall be taken into account the strategic role of new digital technologies and opportunities for successful innovation in the digital world.

M. H. Ismail et al. [28] define digital transformation as the process by which companies integrate different digital technologies, powered by ubiquitous connectivity, with the intention of achieving better performance and competitive resilience by transforming multiple business dimensions, including the business model, customer experience, and business processes (involving digital products and services) simultaneously impacting people (including skills, talents, and culture) and networks (including the entire value system).

Accordingly, DT can be conceptualized at the junction of the introduction of revolutionary digital technologies on the one hand and the organizational transformation of capabilities, structures, processes, and components of a business model, on the other. In other words, DT shall be defined as digital-driven organizational changes (Figure 1).

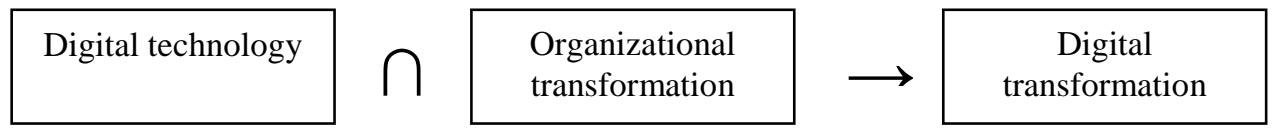

Fig. 1. Digital transformation formula.

\subsection{The paradigm of digital transformation challenges}

Digitalization is a dynamic process where elements of the physical and digital world collide. And those digital initiatives that create new opportunities also lead to new challenges. As a result, there is a constant conflict between the need for digitalization and the need to overcome the challenges that it carries. At that, the nature of these challenges is heterogeneous, multifaceted and interconnected. This contextuality allows us to conclude that the challenges of digital transformation are a set of problems, risks, threats, trends and opportunities caused by digitalization, the complexity and scale of which are such that they cannot be solved, eliminated or implemented solely by increasing resources. Accordingly, an adequate response to these challenges cannot be effectively overcome in an extensive way (due to the lack of available resources or the ineffectiveness of their use for these purposes), since the required resource costs are comparable or exceed the expected effect) and requires qualitative (intensive) changes within the company itself.

At the same time, changes in conditions common for different companies (for example, the structure of the market environment, economic sanctions, customs restrictions, etc.) affect 
their position in different ways, moreover, situations are possible when the same change in conditions for some market participants it means improvement, due to their competitive advantages, and for others - decline. For some companies, the part of the challenges are threats, and for others, opportunities. That is, the interests of different companies in relation to these challenges do not necessarily coincide, and may even be strictly antagonistic, which significantly limits or even excludes the possibilities of cooperation to overcome them.

The DT challenge map contains generalized thematic constructs (problems, risks, threats, trends and opportunities), within which the most frequently encountered challenges are summarized (Fig. 2).

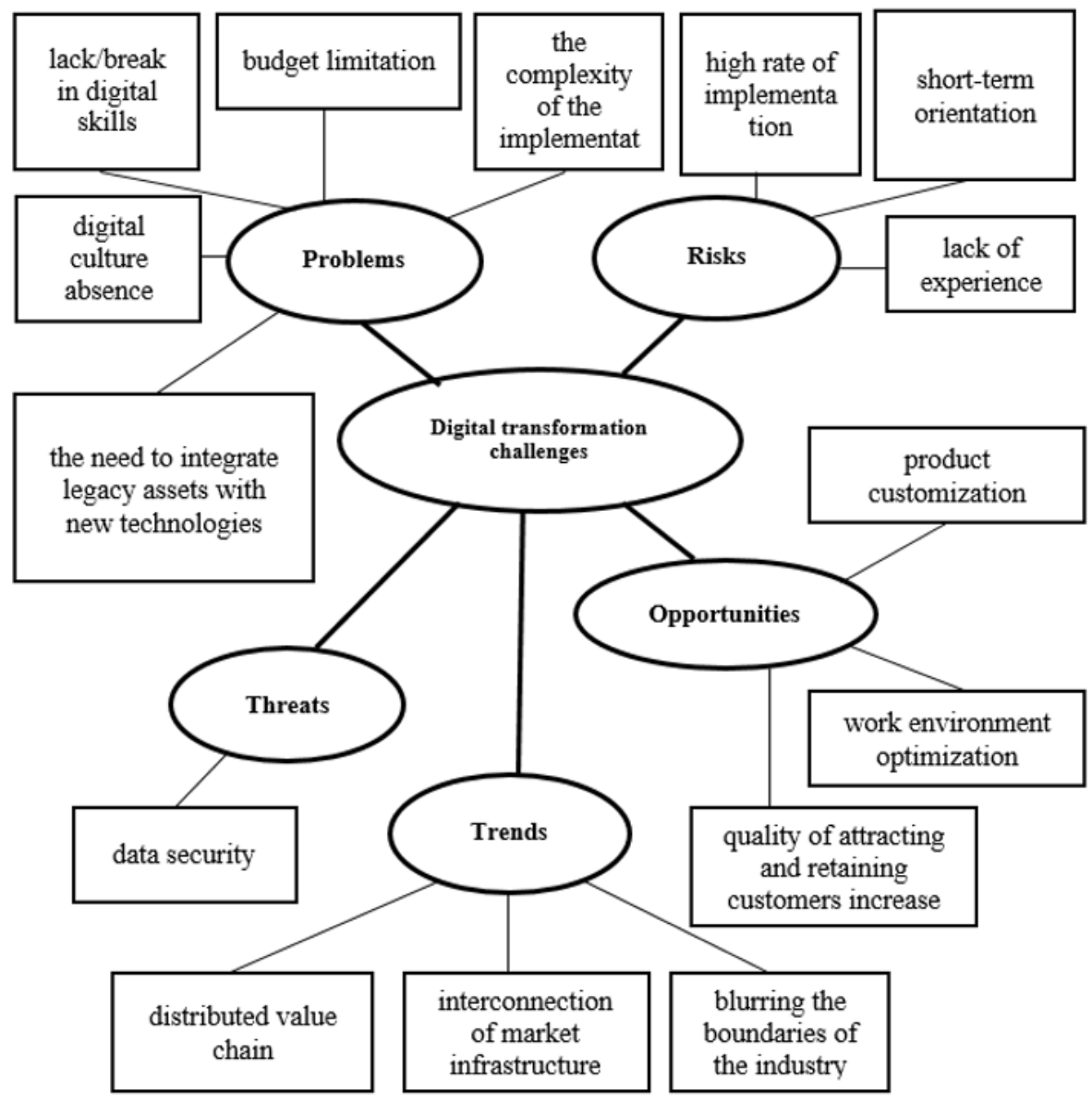

Fig. 2. Digital transformation challenge map.

\section{Problems}

- The need to integrate legacy assets with new technologies

Today, technology integration is ensured by the flexibility of IT, new corporate platforms, and a strong and scalable operating system [20], which is part of the digital infrastructure.

Here raises perhaps the biggest technology challenge for companies moving towards digitalization: how to integrate existing "analog" assets with digital tools. Many companies made significant investments in services that today are not able to create new value and opportunities for business. But giving them up is too expensive. Solving the problem of effectively integrating legacy software within the DT process can repackage the value of 'analog legacy' into vital elements of a digitized company. 
- Digital culture absence

Digital transformation is a technology-driven challenge that requires deep cultural change within a company. Two premises lead to this:

- First, in the digital era, data shall be seen as a valuable resource and a means of achieving the goals of digitalization. In this context, companies need to 'expand' their corporate culture with significant aspects of digital culture [29]. Compared to corporate culture, digital culture values IT as a key element of strategic and tactical decisions and clearly understands the financial and transformative potential of digital technology.

- Secondly, DT can cause cultural conflict between young and relatively inexperienced employees of the digital generation and older, but more experienced employees working before the transition to digital [27].

- Lack/break in digital skills

Another challenge in the context of digitalization challenges is the management of twoway capabilities, in terms of analog and digital skills. Companies have to incorporate 'old' and 'new' skills into their pool of capabilities. It shall be done in such a way that they do not interfere, but complement each other. In the digital world also important networking skills [30], platforms [20], and ecosystems [31]. Depending on contextual factors such as industry or business model, companies must learn to take advantage of network effects in terms of additional opportunities, and learn to transform into an ecosystem rather than continue to manage value chains.

- Budget limitation

When it comes to DT, budget limits are troubling companies around the world. According to a World Economic Forum survey, the cost of digital technologies is the most sharp barrier to their implementation ( $45 \%$ of respondents indicated this).

Sometimes budgets are made at least six months or a year in advance, creating a deficit for promising digital investments. Often, the budgets of companies are consolidated by management, and changing business processes to create new value in the digital realities is associated with limitations and fluctuations in terms of making changes to the budget [33].

- The complexity of the implementation of digital technologies

Digital technologies are constantly generating new tools. And the business keeping at a competitive level requires the constant learning of these new tools. Continuous and complex nature is one of the important problems of DT. Several digital systems/platforms in a company can be incorporated into each other, which is confusing, repressing, destroying targets [33].

Approaching the completion of digital transformation initiatives, many companies make another mistake of not planning on project fatigue[31]. DT projects involve so many people (internal and external, such as system integrators and project management consultants) for such an extended period of time that depletion is inevitable - depletion of staff, depletion of budget, depletion of time.

Risks

- Short term orientation

When companies enter DT, they have to overcome three risks that affect the planning and cost of transformation (Yeo et al., 2018). The first is blindly beginning on digital transformation, the second is the introduction of unnecessary technologies, and the third is the erroneous conclusion that this is a one-time process. And here one of the biggest challenges is to ensure that planning for digital transformation goes beyond planning for the first three to five months. Otherwise, implementation becomes a mere project task that threatens to undermine years of work, millions invested, and organizational breakdown suffered.

- Lack of experience 
Research [5] showed that only $23 \%$ of companies admit they have a strategy of corporate digital transformation. An enormous $77 \%$ of companies are with no overall corporate strategy, focus, or implementation plan. At the same time, $38 \%$ of companies surveyed have a separate business segment or product line that determines the digital transformation of their company.

- High rate of implementation

During the DT period, the rate of technological change accelerates disproportionately, and new digital opportunities emerge every year. The capabilities of end-to-end technologies such as the Internet of Things (IoT), Big Data, cloud computing and mobile technologies are significantly accelerating the overall pace of change. The answer to this should be a faster cycle of strategy development and implementation [33]. A fast and ever-changing digital environment is forcing managers to make decisions and implement strategies much faster than previously required. To study managerial abilities in the context of digital transformation, some studies have adopted the theory of dynamic abilities presented by D. Tees [34].

Digitalization rate is only gaining momentum, and keeping pace with the constantly rolling waves of digital initiatives is another challenge of the transformation period.

\section{Opportunities}

- Product customization

With digitalization, manufacturers can take customer feedback to the next level. It is possible due to hundreds of sensors aggregating data in real time. This dataset provides invaluable information to those responsible for product / service development.

Product customization is a trend that will become more and more important and important for a wider range of companies. Digital technology enable to move to the small batch and mixed production, opening the door to individual product customization. The benefits of this are significant, including increasing revenues, expanding brand reach, and building better customer relationships.

- Work environment optimization

Business digitalization is also changes the day-to-day work environment in companies in terms of work structure, job roles and workplace requirements [24]. Digital collaboration enables flexible and networked teams to be built across multiple locations across a company's geographic map. In this context, traditional hierarchical structures disappear and new opportunities appear outside the company, such as the integration of external freelancers [24]. In addition, the introduction of the digital workplace is becoming inevitable.

- Quality of attracting and retaining customers increase

Customers are behaving differently than before in the new digital marketplace, and traditional marketing methods are becoming ineffective. Today, customers have many ways to easily gather information about products and services well in advance of the actual purchase. In this sense, digital technologies are also transforming the activities of customer interaction strategies [20]. Reaching customers in a digital environment requires digital omnichannel marketing, including social media, mobile apps and augmented reality [31]. In addition, digital technologies are more reducing information asymmetry between buyers and sellers: the availability of information and its ubiquity are fundamentally changing the accepted rules of relations between them. Diversified digital platforms and networks have virtually displaced intermediaries from the analog world.

\section{Threats}

DT's main threats are focused on cybersecurity. And there are several 'benchmarks' here: first, cybernetics (the growing number of devices combined with more sophisticated threat sources means that the risk of a cyberattack is higher than ever); secondly, the use of cloud services (the risks associated with moving or deploying systems to the cloud architecture continue to grow); third, data privacy (controls and regulation of personal information are expanding and, if not managed properly, it can undermine the company's trust, reputation and 
profits). However, security shall be seen not only as a challenge, but also as evidence of the need to accelerate DT initiatives.

Trends

- Distributed value creation

A review of the literature shows that during the DT, the value chain became much more distributed. Two major changes can be observed here: firstly, digital technologies offer customers the opportunity to co-create products with a manufacturer, for example, through digital platforms [31], and secondly, value at the inter-company level is increasingly cocreated and reflected in a series of partnerships in value creation networks [26]. Hence, it can be argued that network effects are a key differentiator and driving force behind the creation and capture of value in the digital world. Thus, the accent in value creation is shifting from the value chain to the value network.

- Blurring the boundaries of the industry

Digital transformation is redefining, blurring and even erasing existing industry boundaries, which can lead, on the one hand, to cross-industry competition (the logic of the dominant industry no longer works, and even new small companies can become serious competitors, and on the other, to digital convergence (convergence of various network platforms during realization of essentially similar types of services). On the supply side, digital convergence is characterized by the use of the same knowledge base by different industries; on the demand side, it erases the boundaries of markets [35].

- Interconnection of market infrastructure

With the advent of digital platforms, networks and ecosystems, market infrastructure becomes more interconnected in a broader sense, there is a transition from control or participation in a linear value chain to work in an ecosystem or network [11]. As different types of innovative networks emerge, with a different cognitive and social translation of knowledge, new digital infrastructure properties shall be required to support each such network. Thus, digital technologies increase the knowledge heterogeneity of innovation networks. However, it should be noted that the free flow of digital products accelerates the destruction of property rights and increases the risk of imitation [20].

\section{Conclusion}

Digital transformation goes far beyond the trend - it is becoming a strategic imperative driven by two collective dimensions: technological transformation and organizational architecture. Within the framework of this concept, a whole system of challenges shall be formed, accepting which, companies undergo a strategic renewal. In turn, the aspect of strategic renewal, also referred to as strategic change, rebirth, transformation [25], reorganization, redefinition [28], or organizational renewal [7], provides a promising interface for digital transformation, the 'ninth wave' of which has already covered many industry 'headlong'. And here it is necessary to clearly understand that DT waves are not an isolated event, but should be viewed as a new norm that requires companies to constantly change, develop and innovate.

\section{References}

1. G. Kane, MIT Sloan Management Review 58(2), 17 (2017)

2. I. Kaufman, C. Horton, The European Financial Review (Dec.-Jan.), 63 (2015)

3. M. Fitzgerald, MIT Sloan Management Review, 1 (2013)

4. E.I. Schwartz, Web Economy, Broadway (2001) 
5. Realizing the digital promise. Deloitte. https://www2.deloitte.com/content/dam/Deloitte/global/Documents/FinancialServices/gx-fsi-iif-deloitte-digital-transformation-study-2020-report.pdf

6. Unlocking success in digital transformations. McKinsey. https://www.mckinsey.com/business-functions/organization/our-insights/unlockingsuccess-in-digital-transformations

7. C. Matt, T. Hess, A. Benlian, Business \& Information Systems Engineering 57(5), 339 (2014)

8. T. Hess, MIS Quarterly Executive 15(2), 123 (2016)

9. N. Kulatilaka, N.Venkatraman, Ebusiness, 26 (2015)

10. K. Schwab, World Economic Forum (2016)

11. J. Anderson, G. Lanzolla, Business Strategy Review 21(1), 74 (2010)

12. R. Atkinson, Economic Development Journal, Summer, 33 (2005)

13. R.C. Basole, IT Pro (December), 20 (2016)

14. D. Muzyka, European Management Journal 13(4), 346 (1995)

15. C.K. Prahalad, J.P., Oosterveld, Sloan Management Review 42(2), 31 (1999)

16. V.J Richardson, B.Dehning. MIS Quarterly, 27(4), 637 (2003)

17. F. Gouillart, J.Kelly, Transforming the Organization, 1995.

18. R.E. Morgan, K. Page, Strategic Change 17, 155 (2008)

19. G.C. Kane, MIT Sloan Management Review, 1 (2015)

20. I.M. Sebastian, MIS Quarterly Executive, 16(3), 197 (2017)

21. T. Jelassi, S. Dutta, Journal of Strategic Information Systems, 2(1), 77 (1993)

22. P.W., Yetton, K.D. Johnston, Sloan Management Review (summer), 57 (1994)

23. S.J. Andriole, MIT Sloan Management Review, 58(3), 20 (2017)

24. N. Webb, Human Resource Management International Digest 21(1), 5 (2013)

25. G. Westerman, MIT Sloan Management Review, 16 (2012)

26. M. Chahal, Marketing Week (April), 16 (2016)

27. R Kohli, S. Johnson, MIS Quarterly Executive, 10(4), 412 (2011)

28. M. H. Ismail, M. Khater, M. Zaki, Cambridge Service Alliance 2017, 35 (2019)

29. J. Llopis, M. Gonzalez, J. Gasco, Hum. Syst. Manag. 23(4), 213 (2004)

30. A. Bharadwaj, O. El Sawy, P. Pavlou, MIS Q 37, 471 (2013)

31. O. El Sawy, A. Malhotra, Inf. Syst. Res. 21(4), 835 (2010)

32. S. Nadkarni, R. Prügl, Management Review Quarterly 4, 233 (2020)

33. E. Daniel, H. Wilson, Eur. J. Inf. Syst. 12, 282 (2003)

34. D. Teece, J. Int. Bus. Stud. 45(1), 8 (2014)

35. B. Kaluza, T. Blecker, C. Bischof, The Current State of Economic Science 4, 2223 (1999). 